\title{
ON THE FORCED SURFACE WAVES DUE TO A PARTIALLY IMMERSED VERTICAL WAVE-MAKER IN WATER OF INFINITE DEPTH
}

\author{
$\mathrm{BY}$ \\ P. F. RHODES-ROBINSON \\ Department of Mathematics, Victoria University of Wellington, New Zealand
}

\begin{abstract}
In this paper we determine the velocity potential describing the twodimensional antisymmetric wave motion generated by a harmonically oscillating vertical plane wave-maker partially immersed in water of infinite depth. The solution of the boundary-value problem for the velocity potential is obtained from a formulation depending on the unknown horizontal velocity on the line of antisymmetry below the wave-maker, which leads to a singular integral equation of known type for a related reduction variable. The idea was developed by Ursell to solve the corresponding transmission problem and another rigid wave-maker problem, and uses classical wave-maker theory.
\end{abstract}

1. Introduction. The classical wave-maker theory of Havelock [3] to determine the velocity potential for two-dimensional time-harmonic wave motion in water of infinite depth in the absence of surface tension is well known. The wave-maker boundary, on which a simple-harmonic normal velocity distribution is prescribed, is vertical and extends throughout the depth of water, and the motion has the form of outgoing waves at infinite horizontal distance.

Herein we consider the motion produced in water of infinite depth and expanse when the wave-maker is only partially immersed with its lower end at a given finite depth below the free surface. The motion is antisymmetric, with outgoing waves at infinite horizontal distance in both directions. The motion due to one such wave-maker comprising a rigid vertical plate oscillating about a horizontal axis in itself was determined by Ursell [6], who previously had also found the scattering effect on incoming waves by a partially immersed fixed vertical plane barrier-Ursell [5].

The method developed in Ursell [5], [6] is extended herein. The velocity potential is given by the classical wave-maker theory of Havelock [3] if the unknown horizontal velocity on the line of antisymmetry below the wave-maker can be determined. This unknown satisfies a complicated integral equation that is solved by reduction to a singular integral equation for a related variable, upon which the required velocity potential can in fact be made to depend, to complete the solution most expediently.

Received July 12, 1994.

1991 Mathematics Subject Classification. Primary 76B15.

(C)1996 Brown University 
The technique involves real variables and is suitable only for two-dimensional motion on infinite depth; it may be amenable to the inclusion of surface tension, but this effect is omitted herein. Complex-variable techniques that do not readily yield a velocity potential were employed by Haskind [2], who effectively combined the two problems in Ursell [5], [6]; and by Lewin [4], who made a formal investigation of incomplete vertical wave-maker problems in general.

The outgoing waves in our problem have been determined in another form by Evans [1] using the real-variable technique of exact Green's functions, provided in this case by the transmission solution of Ursell [5].

2. Formulation and reduction of problem. Two-dimensional time-harmonic wave motion of angular frequency $\sigma$ is set up in an ideal liquid of infinite depth and expanse by small prescribed normal oscillations of a vertical plane wave-maker that pierces the free surface and is partially immersed to a depth $a$. The motion is antisymmetric about the gap below the wave-maker, and is assumed to be small with outgoing surface waves at infinity. The effect of surface tension is excluded, and the motion is under the action of gravity $g$ alone. In terms of rectangular coordinates $x, y$ the velocity potential at time $t$ has the steady-state form $\operatorname{re}\left[\phi(x, y) e^{-i \sigma t}\right]$, where $\phi$ is complex-valued and satisfies a boundary-value problem in the liquid region. It is sufficient to consider only the quarter-plane $y>0, x>0$ bounded by the free surface $y=0$, wave-maker $x=0,0<y<a$, and line of antisymmetry $x=0, y>a$ below it: then, if the horizontal velocity on the wave-maker into this region is $\mathrm{re}\left[U_{0}(y) e^{-i \sigma t}\right]$ and a flat-plate type infinite velocity singularity is allowed at the tip of the wave-maker, $\phi$ satisfies the equation

$$
\nabla^{2} \phi=0 \quad \text { in } x>0, y>0
$$

subject to the boundary conditions

$$
\begin{aligned}
& K \phi+\phi_{y}=0 \text { on } y=0, \\
& \phi_{x}=U_{0}(y) \quad \text { on } x=0,0<y<a \text {, } \\
& \phi=0 \quad \text { on } x=0, y>a, \\
& r^{\frac{1}{2}}|\nabla \phi| \text { is bounded as } r \equiv\left[x^{2}+(y-a)^{2}\right]^{\frac{1}{2}} \rightarrow 0 \text {, } \\
& |\nabla \phi| \rightarrow 0 \text { as } y \rightarrow \infty, \\
& \phi \rightarrow \text { a multiple of } e^{-K y+i K x} \text { as } x \rightarrow \infty \text {, }
\end{aligned}
$$

where $K=\sigma^{2} / g$.

This boundary-value problem may be solved by classical wave-maker theory if the unknown horizontal velocity $\phi_{x}=U(y)$, say, on $x=0, y>a$ can be found satisfying the conditions that $(y-a)^{\frac{1}{2}}|U|$ is bounded as $y \rightarrow a+$ and $|U| \rightarrow 0$ as $y \rightarrow \infty$. For then, by Green's theorem, the solution is given by the distribution

$$
\phi(x, y)=\frac{1}{\pi} \int_{0}^{\infty} G(x, y ; Y) U(Y) d Y
$$


where $U=U_{0}(0<Y<a)$ is already known and $G(x, y ; Y)$ is the well-known potential for a wave source at $(0, Y)$; this is given by the alternative forms

$$
G(x, y ; Y)=\log \frac{\rho}{\rho^{\prime}}-2 \psi_{0}^{\infty} \frac{e^{-k(y+Y)}}{k-K} \cos k x d k
$$

where $\rho, \rho^{\prime}=\left[x^{2}+(y \mp Y)^{2}\right]^{\frac{1}{2}}$ respectively and the contour is indented below the pole $k=K$, and

$$
\begin{gathered}
G(x, y ; Y)=-2 \int_{0}^{\infty} \frac{e^{-k x}}{k\left(k^{2}+K^{2}\right)}(k \cos k y-K \sin k y)(k \cos k Y-K \sin k Y) d k \\
-2 \pi i e^{-K(y+Y)+i K x} .
\end{gathered}
$$

Explicitly, the wave-maker solution $(2.1)$ is

$$
\phi(x, y)=-\frac{2}{\pi} \int_{0}^{\infty} \frac{a(k) e^{-k x}}{k^{2}+K^{2}}(k \cos k y-K \sin k y) d k-2 i A e^{-K y+i K x}
$$

from (2.3), where

$$
a(k)=\frac{1}{k} \int_{0}^{\infty} U(Y)(k \cos k Y-K \sin k Y) d Y \quad(k>0)
$$

and

$$
A=\int_{0}^{\infty} U(Y) e^{-K Y} d Y
$$

in the original form obtained by Havelock [3]; the first term is non-propagating and the second term represents the outgoing waves at infinity.

An integral equation is obtained for the unknown $U(Y>a)$ by applying the remaining antisymmetry condition: hence

$$
\phi(0, y)=\int_{0}^{\infty} G^{*}(y ; Y) U(Y) d Y=0 \quad(y>a)
$$

where $G^{*}(y ; Y)=G(0, y ; Y)$. This is simplified, following the approach of Ursell [5], [6], by applying a differential operator suggested by the free surface condition; thus, from (2.7)

$$
\left(K+\frac{d}{d y}\right) \int_{0}^{\infty} G^{*}(y ; Y) U(Y) d Y=0
$$

and so

$$
f_{0}^{\infty}\left[K G^{*}(y ; Y)+G_{y}^{*}(y ; Y)\right] U(Y) d Y=0 \quad(y>a)
$$

where the Cauchy principal value (CPV) is needed at $Y=y$. But

$$
G^{*}=\log \frac{|y-Y|}{y+Y}-2 \psi_{0}^{\infty} \frac{e^{-k(y+Y)}}{k-K} d k
$$


from (2.2), and so

$$
K G^{*}+G_{y}^{*}=K \log \frac{|y-Y|}{y+Y}+\frac{1}{y-Y}-\frac{1}{y+Y}+\frac{2}{y+Y}=K \log \frac{|y-Y|}{y+Y}+\frac{2 y}{y^{2}-Y^{2}}
$$

thus

$$
K \int_{0}^{\infty} U(Y) \log \frac{|y-Y|}{y+Y} d Y+2 y f_{0}^{\infty} \frac{U(Y)}{y^{2}-Y^{2}} d Y=0 \quad(y>a)
$$

Now let

$$
F(Y)=\int_{0}^{Y} U(s) d s \quad(Y>0)
$$

so that $F^{\prime}=U$ and $F(0)=0$; then

$$
F=F_{0} \equiv \int_{0}^{Y} U_{0}(s) d s \quad(0<Y<a)
$$

say, is known and $F_{0}(0)=0$. Therefore

$$
\int_{0}^{\infty} U(Y) \log \frac{|y-Y|}{y+Y} d Y=2 y f_{0}^{\infty} \frac{F(Y)}{y^{2}-Y^{2}} d Y
$$

by integration by parts.

Hence (2.7) reduces to

$$
f_{a}^{\infty} \frac{g(Y)}{y^{2}-Y^{2}} d Y=0 \quad(y>a)
$$

where $g=K F+U=K F+F^{\prime}$ is a reduction variable, and so we obtain the simplified integral equation

$$
f_{a}^{\infty} \frac{g(Y)}{y^{2}-Y^{2}} d Y=-\int_{0}^{a} \frac{g_{0}(Y)}{y^{2}-Y^{2}} d Y \quad(y>a)
$$

for the unknown $g(Y>a)$ in terms of the known $g=g_{0} \equiv K F_{0}+U_{0}(0<Y<a)$, say. Note that $(Y-a)^{\frac{1}{2}}|g|$ must be bounded as $Y \rightarrow a+$ and $|g|$ must be bounded as $Y \rightarrow \infty$.

It is possible - and obviously convenient - to rephrase the wave-maker solution (2.4) in terms of $g(Y)$ also rather than $U(Y)$. Thus, from (2.5)

$$
a(k)=\frac{1}{k} \int_{0}^{\infty} F^{\prime}(Y)(k \cos k Y-K \sin k Y) d Y=\int_{0}^{\infty}[g(Y)-g(\infty)] \cos k Y d Y \quad(k>0)
$$

after integration by parts, where $g(\infty)=\lim _{Y \rightarrow \infty} g(Y)$; and similarly from $(2.6)$

$$
A=\int_{0}^{\infty} F^{\prime}(Y) e^{-K Y} d Y=\frac{1}{2} \int_{0}^{\infty} g(Y) e^{-K Y} d Y
$$

since $F(0)=0$. These replace $(2.5,2.6)$, and in them $g(Y>a)$ is to be found and $g=g_{0}$ $(0<Y<a)$ is known. 
3. Solution of integral equation. Nonhomogeneous singular integral equations of the above type were solved by Ursell [5], [6]. The formal solution of $(2.8)$ for $g(y>a)$ is given by

$$
\frac{\left(y^{2}-a^{2}\right)^{\frac{1}{2}}}{y} g(y)=D-\frac{4 y^{2}}{\pi^{2}} f_{a}^{\infty} \frac{\left(Y^{2}-a^{2}\right)^{\frac{1}{2}}}{Y\left(Y^{2}-y^{2}\right)} \int_{0}^{a} \frac{g_{0}(s)}{Y^{2}-s^{2}} d s d Y,
$$

where the outer CPV is at $Y=y$ and $D$ is an arbitrary constant,

$$
=D-\frac{4 y^{2}}{\pi^{2}} \int_{0}^{a} g_{0}(s) f_{a}^{\infty} \frac{\left(Y^{2}-a^{2}\right)^{\frac{1}{2}}}{Y\left(Y^{2}-y^{2}\right)\left(Y^{2}-s^{2}\right)} d Y d s
$$

on interchanging the order of integration

$$
=D+\frac{2}{\pi} \int_{0}^{a} g_{0}(s)\left[\frac{a-\left(a^{2}-s^{2}\right)^{\frac{1}{2}}}{s^{2}}-\frac{\left(a^{2}-s^{2}\right)^{\frac{1}{2}}}{y^{2}-s^{2}}\right] d s
$$

on evaluating the inner CPV integral (see Appendix 2a)

$$
=D^{\prime}-\frac{2}{\pi} \int_{0}^{a} g_{0}(s) \frac{\left(a^{2}-s^{2}\right)^{\frac{1}{2}}}{y^{2}-s^{2}} d s
$$

finally, where

$$
D^{\prime}=D+\frac{2}{\pi} \int_{0}^{a} g_{0}(s) \frac{a-\left(a^{2}-s^{2}\right)^{\frac{1}{2}}}{s^{2}} d s
$$

is now taken as the arbitrary constant.

Note that the required boundedness conditions on $g(y>a)$ are satisfied by (3.2), since

$$
(y-a)^{\frac{1}{2}} g(y) \rightarrow\left(\frac{1}{2} a\right)^{\frac{1}{2}}\left[D^{\prime}-\frac{2}{\pi} \int_{0}^{a} \frac{g_{0}(s)}{\left(a^{2}-s^{2}\right)^{\frac{1}{2}}} d s\right] \quad \text { as } y \rightarrow a^{+}
$$

and

$$
g(y) \rightarrow D^{\prime}=g(\infty) \quad \text { as } y \rightarrow \infty .
$$

4. Calculation of wave-maker solution. The results incorporating (3.2)

$$
\begin{aligned}
g(Y) & =g_{0}(Y) \quad(0<Y<a) \\
& =\frac{Y}{\left(Y^{2}-a^{2}\right)^{\frac{1}{2}}}\left[D^{\prime}-\frac{2}{\pi} \int_{0}^{a} g_{0}(s) \frac{\left(a^{2}-s^{2}\right)^{\frac{1}{2}}}{Y^{2}-s^{2}} d s\right] \quad(Y>a)
\end{aligned}
$$

are now used to evaluate $(2.9,2.10)$ in terms of $D^{\prime}$.

First, we have from (2.9) that for $k>0$

$$
a(k)=a_{0}(k)+a_{1}(k)
$$


say, where

$$
a_{0}(k)=\int_{0}^{a} g_{0}(Y) \cos k Y d Y
$$

and

$$
\begin{aligned}
& a_{1}(k)= \int_{a}^{\infty}[g(Y)-g(\infty)] \cos k Y d Y-g(\infty) \frac{\sin k a}{k} \\
&= \int_{a}^{\infty} \cos k Y\left[D^{\prime} \frac{Y-\left(Y^{2}-a^{2}\right)^{\frac{1}{2}}}{\left(Y^{2}-a^{2}\right)^{\frac{1}{2}}}-\frac{2 Y}{\pi\left(Y^{2}-a^{2}\right)^{\frac{1}{2}}} \int_{0}^{a} g_{0}(s) \frac{\left(a^{2}-s^{2}\right)^{\frac{1}{2}}}{Y^{2}-s^{2}} d s\right] d Y \\
& \quad-D^{\prime} \frac{\sin k a}{k} \\
&=D^{\prime} {\left[\int_{a}^{\infty} \frac{Y-\left(Y^{2}-a^{2}\right)^{\frac{1}{2}}}{\left(Y^{2}-a^{2}\right)^{\frac{1}{2}}} \cos k Y d Y-\frac{\sin k a}{k}\right] } \\
& \quad-\frac{2}{\pi} \int_{0}^{a} g_{0}(s)\left(a^{2}-s^{2}\right)^{\frac{1}{2}} \int_{a}^{\infty} \frac{Y \cos k Y}{\left(Y^{2}-a^{2}\right)^{\frac{1}{2}}\left(Y^{2}-s^{2}\right)} d Y d s \\
&=-\frac{1}{2} \pi a D^{\prime} J_{1}(k a)-\int_{0}^{a} g_{0}(s)\left[\cos k s-\left(a^{2}-s^{2}\right)^{\frac{1}{2}} \int_{0}^{k} \cos (k-u) s J_{0}(u a) d u\right] d s
\end{aligned}
$$

on noting the integral representation of a Bessel function (see Appendix 1a) and evaluating another integral in convolution form involving another Bessel function (see Appendix 2b). Hence

$$
a(k)=-\frac{1}{2} \pi a D^{\prime} J_{1}(k a)+\int_{0}^{k} b(k-u) J_{0}(u a) d u \quad(k>0)
$$

after a cancellation, where

$$
b(u)=\int_{0}^{a} g_{0}(s)\left(a^{2}-s^{2}\right)^{\frac{1}{2}} \cos u s d s \quad(u>0) .
$$

Second, we have from (2.10) that

$$
A=A_{0}+A_{1}
$$

say, where

$$
A_{0}=\frac{1}{2} \int_{0}^{a} g_{0}(Y) e^{-K Y} d Y
$$

and

$$
\begin{aligned}
A_{1} & =\frac{1}{2} \int_{a}^{\infty} g(Y) e^{-K Y} d Y \\
& =\frac{1}{2} \int_{a}^{\infty} \frac{Y e^{-K Y}}{\left(Y^{2}-a^{2}\right)^{\frac{1}{2}}}\left[D^{\prime}-\frac{2}{\pi} \int_{0}^{a} g_{0}(s) \frac{\left(a^{2}-s^{2}\right)^{\frac{1}{2}}}{Y^{2}-s^{2}} d s\right] d Y \\
& =\frac{1}{2} D^{\prime} \int_{a}^{\infty} \frac{Y e^{-K Y}}{\left(Y^{2}-a^{2}\right)^{\frac{1}{2}}} d Y-\frac{1}{\pi} \int_{0}^{a} g_{0}(s)\left(a^{2}-s^{2}\right)^{\frac{1}{2}} \int_{a}^{\infty} \frac{Y e^{-K Y}}{\left(Y^{2}-a^{2}\right)^{\frac{1}{2}}\left(Y^{2}-s^{2}\right)} d Y d s \\
& =\frac{1}{2} a D^{\prime} K_{1}(K a)-\frac{1}{\pi} \int_{0}^{a} g_{0}(s)\left[\frac{1}{2} \pi e^{-K s}+\sinh K s \cos ^{-1} \frac{s}{a}\right. \\
& \left.-\int_{0}^{K} \cosh (K-u) s K_{0}(u a) d u\right] d s
\end{aligned}
$$


on noting the integral representation of a modified Bessel function (see Appendix 1b) and evaluating another integral in convolution form involving another modified Bessel function (see Appendix 2c). Hence

$$
A=\frac{1}{2} a D^{\prime} K_{1}(K a)-\frac{1}{\pi} B+\frac{1}{\pi} \int_{0}^{K} c(K-u) K_{0}(u a) d u
$$

after a cancellation, where

$$
B=\int_{0}^{a} g_{0}(s) \sinh K s \cos ^{-1} \frac{s}{a} d s
$$

and

$$
c(u)=\int_{0}^{a} g_{0}(s)\left(a^{2}-s^{2}\right)^{\frac{1}{2}} \cosh u s d s \quad(u>0) .
$$

To complete the solution (2.4), the integration constant $D^{\prime}$ is determined from the linear equation obtained by substituting the values $(4.1,4.3)$ into the original integral equation (2.7) taken in the explicit form

$$
-\phi(0, y)=\frac{2}{\pi} \int_{0}^{\infty} \frac{a(k)}{k^{2}+K^{2}}(k \cos k y-K \sin k y) d k+2 i A e^{-K y}=0 \quad(y>a),
$$

using (2.4). Hence

$$
-\frac{1}{2} \pi a D^{\prime} I^{(1)}(y)+I^{(2)}(y)+i e^{-K y}\left[\frac{1}{2} \pi a D^{\prime} K_{1}(K a)-B+\int_{0}^{K} c(K-u) K_{0}(u a) d u\right]=0
$$

$(y>a)$, where

$$
\begin{aligned}
I^{(1)} & =\int_{0}^{\infty} \frac{J_{1}(k a)}{k^{2}+K^{2}}(k \cos k y-K \sin k y) d k \\
& =\frac{1}{2} \int_{-\infty}^{\infty} \frac{J_{1}(k a)}{k-i K} e^{i k y} d k \quad(y>a) \\
& =-\pi e^{-K y} I_{1}(K a)
\end{aligned}
$$

by the method of residues at the pole $k=i K$; and

$$
\begin{aligned}
I^{(2)} & =\int_{0}^{\infty} \frac{k \cos k y-K \sin k y}{k^{2}+K^{2}} \int_{0}^{k} b(k-u) J_{0}(u a) d u d k \\
& =\int_{0}^{1} \int_{0}^{\infty} b[k(1-v)] \frac{k J_{0}(k a v)}{k^{2}+K^{2}}(k \cos k y-K \sin k y) d k d v \quad(u=k v) \\
& =\int_{0}^{1} \int_{0}^{a} g_{0}(s)\left(a^{2}-s^{2}\right)^{\frac{1}{2}} I^{(3)}(y, s ; v) d s d v
\end{aligned}
$$

from (4.2), where

$$
\begin{aligned}
I^{(3)} & =\int_{0}^{\infty} \frac{k J_{0}(k a v)}{k^{2}+K^{2}}(k \cos k y-K \sin k y) \cos k(1-v) s d k \quad(0<s<a<y, 0<v<1) \\
& =\frac{1}{2} \int_{-\infty}^{\infty} \frac{k J_{0}(k a v)}{k-i K} e^{i k y} \cos k(1-v) s d k \quad[y-(1-v) s>a v] \\
& =-\pi K e^{-K y} \cosh K(1-v) s I_{0}(K a v)
\end{aligned}
$$


by the method of residues at the pole $k=i K$, so that

$$
\begin{aligned}
I^{(2)} & =-\pi K e^{-K y} \int_{0}^{1} c[K(1-v)] I_{0}(K a v) d v \\
& =-\pi e^{-K y} \int_{0}^{K} c(K-u) I_{0}(u a) d u \quad(u=K v)
\end{aligned}
$$

from (4.5).

Hence, using these and cancelling a factor $e^{-K y}, D^{\prime}$ is given by

$$
\frac{1}{2} \pi a\left[\pi I_{1}(K a)+i K_{1}(K a)\right] D^{\prime}=i B+\int_{0}^{K} c(K-u)\left[\pi I_{0}(u a)-i K_{0}(u a)\right] d u
$$

so that the solution is complete. This is extremely complicated to apply theoretically, even in simple cases, but computational possibilities exist.

Note, using $(4.3,4.6)$, that $A$ is given by

$\left[\pi I_{1}(K a)+i K_{1}(K a)\right] A=-B I_{1}(K a)+\int_{0}^{K} c(K-u)\left[K_{1}(K a) I_{0}(u a)+I_{1}(K a) K_{0}(u a)\right] d u$ for the outgoing waves. A simple result to determine these (only) was obtained by Evans [1], using the exact Green's function in the far field-viz. the transmission solution for a partially immersed fixed barrier in Ursell [5]. In our notation, this is

$$
\begin{aligned}
& {\left[\pi I_{1}(K a)+i K_{1}(K a)\right] A} \\
& \quad=\frac{1}{a} \int_{0}^{a} \frac{Y e^{K Y}}{\left(a^{2}-Y^{2}\right)^{\frac{1}{2}}} \int_{0}^{Y} U_{0}(s) e^{-K s} d s d Y \\
& \quad=\frac{1}{2 a} \int_{0}^{a} \frac{Y}{\left(a^{2}-Y^{2}\right)^{\frac{1}{2}}}\left[e^{K Y} \int_{0}^{Y} g_{0}(s) e^{-K s} d s+e^{-K Y} \int_{0}^{Y} g_{0}(s) e^{K s} d s\right] d Y
\end{aligned}
$$

in terms of $g_{0}$ used herein (see Appendix 3).

\section{Appendices.}

1. Integral representation of Bessel functions.

a. For $z>0$

$$
J_{0}(z)=\frac{2}{\pi} \int_{1}^{\infty} \frac{\sin \beta z}{\left(\beta^{2}-1\right)^{\frac{1}{2}}} d \beta
$$

and

$$
J_{1}(z)=\frac{2}{\pi}\left[\frac{\sin z}{z}-\int_{1}^{\infty} \frac{\beta-\left(\beta^{2}-1\right)^{\frac{1}{2}}}{\left(\beta^{2}-1\right)^{\frac{1}{2}}} \cos \beta z d \beta\right]
$$

the second result is obtained from the first by careful differentiation, although an interesting proof was given by Ursell [5].

b.

$$
K_{0}(z)=\int_{1}^{\infty} \frac{e^{-\beta z}}{\left(\beta^{2}-1\right)^{\frac{1}{2}}} d \beta
$$

and

$$
K_{1}(z)=\int_{1}^{\infty} \frac{\beta e^{-\beta z}}{\left(\beta^{2}-1\right)^{\frac{1}{2}}} d \beta
$$


2. Evaluation of integrals.

a. The integral

$$
\begin{aligned}
f_{a}^{\infty} & \frac{\left(Y^{2}-a^{2}\right)^{\frac{1}{2}}}{Y\left(Y^{2}-y^{2}\right)\left(Y^{2}-s^{2}\right)} d Y \quad(0<s<a<y) \\
& =f_{0}^{\infty} \frac{z^{2}}{\left[z^{2}-\left(y^{2}-a^{2}\right)\right]\left(z^{2}+a^{2}-s^{2}\right)\left(z^{2}+a^{2}\right)} d z \quad\left[z=\left(Y^{2}-a^{2}\right)^{\frac{1}{2}}\right] \\
& =\frac{1}{y^{2} s^{2}\left(y^{2}-s^{2}\right)} f_{0}^{\infty}\left[\frac{s^{2}\left(y^{2}-a^{2}\right)}{z^{2}-\left(y^{2}-a^{2}\right)}+\frac{y^{2}\left(a^{2}-s^{2}\right)}{z^{2}+a^{2}-s^{2}}-\frac{a^{2}\left(y^{2}-s^{2}\right)}{z^{2}+a^{2}}\right] d z
\end{aligned}
$$

by partial fractions

$$
=\frac{\pi}{2 y^{2}}\left[\frac{y^{2}\left(a^{2}-s^{2}\right)^{\frac{1}{2}}}{s^{2}\left(y^{2}-s^{2}\right)}-\frac{a}{s^{2}}\right]=\frac{\pi}{2 y^{2}}\left[\frac{\left(a^{2}-s^{2}\right)^{\frac{1}{2}}}{y^{2}-s^{2}}-\frac{a-\left(a^{2}-s^{2}\right)^{\frac{1}{2}}}{s^{2}}\right]
$$

by elementary integration and rearrangement.

b. The integral

$$
\int_{a}^{\infty} \frac{Y \cos k Y}{\left(Y^{2}-a^{2}\right)^{\frac{1}{2}}\left(Y^{2}-s^{2}\right)} d Y \quad(0<s<a, k>0)
$$

has Laplace transform in $k$ with parameter $p$ given by

$$
\begin{aligned}
\int_{a}^{\infty} & \frac{Y}{\left(Y^{2}-a^{2}\right)^{\frac{1}{2}}\left(Y^{2}-s^{2}\right)} \frac{p}{Y^{2}+p^{2}} d Y \\
& =p \int_{0}^{\infty} \frac{1}{\left(z^{2}+a^{2}-s^{2}\right)\left(z^{2}+a^{2}+p^{2}\right)} d z \quad\left[z=\left(Y^{2}-a^{2}\right)^{\frac{1}{2}}\right] \\
& =\frac{p}{p^{2}+s^{2}} \int_{0}^{\infty}\left[\frac{1}{z^{2}+a^{2}-s^{2}}-\frac{1}{z^{2}+a^{2}+p^{2}}\right] d z \\
& =\frac{1}{2} \frac{\pi p}{p^{2}+s^{2}}\left[\frac{1}{\left(a^{2}-s^{2}\right)^{\frac{1}{2}}}-\frac{1}{\left(a^{2}+p^{2}\right)^{\frac{1}{2}}}\right]
\end{aligned}
$$

similarly as above, and so by inversion has the value

$$
\frac{1}{2} \pi\left[\frac{\cos k s}{\left(a^{2}-s^{2}\right)^{\frac{1}{2}}}-\int_{0}^{k} \cos (k-u) s J_{0}(u a) d u\right]
$$

c. The integral

$$
\begin{aligned}
& \int_{a}^{\infty} \frac{Y e^{-K Y}}{\left(Y^{2}-a^{2}\right)^{\frac{1}{2}}\left(Y^{2}-s^{2}\right)} d Y \quad(0<s<a) \\
& \quad=\int_{a}^{\infty} \frac{1}{\left(Y^{2}-a^{2}\right)^{\frac{1}{2}}} \int_{K}^{\infty} e^{-u Y} \cosh (u-K) s d u d Y
\end{aligned}
$$


using a simple integral representation,

$$
\begin{aligned}
& =\int_{K}^{\infty} \cosh (u-K) s \int_{a}^{\infty} \frac{e^{-u Y}}{\left(Y^{2}-a^{2}\right)^{\frac{1}{2}}} d Y d u \\
& =\int_{K}^{\infty} \cosh (u-K) s K_{0}(u a) d u
\end{aligned}
$$

on noting the integral representation of a modified Bessel function (see Appendix 1b)

$$
=\frac{1}{2} \frac{\pi e^{-K s}}{\left(a^{2}-s^{2}\right)^{\frac{1}{2}}}+\frac{\sinh K s \cos ^{-1} \frac{s}{a}}{\left(a^{2}-s^{2}\right)^{\frac{1}{2}}}-\int_{0}^{K} \cosh (K-u) s K_{0}(u a) d u,
$$

using the results

$$
\int_{0}^{\infty} \cosh u s K_{0}(u a) d u=\frac{1}{2} \frac{\pi}{\left(a^{2}-s^{2}\right)^{\frac{1}{2}}}
$$

and

$$
\int_{0}^{\infty} \sinh u s K_{0}(u a) d u=\frac{\frac{1}{2} \pi-\cos ^{-1} \frac{s}{a}}{\left(a^{2}-s^{2}\right)^{\frac{1}{2}}} \quad(0<s<a) .
$$

3. Outgoing wave forms. The form for $A$ as obtained by Evans [1] is a multiple of

$$
\int_{0}^{a} \frac{Y f(Y)}{\left(a^{2}-Y^{2}\right)^{\frac{1}{2}}} d Y
$$

where

$$
f(Y)=e^{K Y} \int_{0}^{Y} U_{0}(s) e^{-K s} d s \quad(0<Y<a)
$$

in terms of $U_{0}$.

To express this in terms of $g_{0}$, first note that $f(Y)$ is the solution of the differential equation

$$
f^{\prime}-K f=U_{0} \quad \text { in } 0<Y<a
$$

subject to the condition $f(0)=0$. Now let

$$
G(Y)=\int_{0}^{Y} f(s) d s \quad(0<Y<a)
$$

so that $G^{\prime}=f$ and $G(0)=0$; then term-by-term integration in (A3.1) gives

$$
G^{\prime}-K G=F_{0} \text { for } 0<Y<a .
$$

Multiply (A3.2) by $K$ and add to (A3.1); thus $G$ satisfies the differential equation

$$
G^{\prime \prime}-K^{2} G=g_{0} \quad \text { in } 0<Y<a
$$

subject to the conditions $G(0)=G^{\prime}(0)=0$. The solution is

$$
G(Y)=\frac{1}{K} \int_{0}^{Y} g_{0}(s) \sinh K(Y-s) d s
$$

and so by differentiation

$$
\begin{aligned}
f(Y) & =\int_{0}^{Y} g_{0}(s) \cosh K(Y-s) d s \\
& =\frac{1}{2}\left[e^{K Y} \int_{0}^{Y} g_{0}(s) e^{-K s} d s+e^{-K Y} \int_{0}^{Y} g_{0}(s) e^{K s} d s\right] \quad(0<Y<a)
\end{aligned}
$$

in terms of $g_{0}$. 


\section{REFERENCES}

[1] D. V. Evans, A note on the waves produced by the small oscillations of a partially immersed vertical plate, J. Inst. Math. Appl. 17, 135-140 (1976)

[2] M. D. Haskind, The radiation and diffraction of surface waves from a vertically floating plate, Prikl. Mat. Meh. 23, 546-556 (1959) (Russian), translated as J. Appl. Math. Mech. 23, 770-783 (1960)

[3] T. H. Havelock, Forced surface waves on water, Philos. Mag. 8, 569-576 (1929)

[4] M. Lewin, The effect of vertical barriers on progressing waves, J. Math. and Phys. 42, 287-300 (1963)

[5] F. Ursell, The effect of a fixed vertical barrier on surface waves in deep water, Proc. Cambridge Philos. Soc. 43, 374-382 (1947)

[6] F. Ursell, On the waves due to the rolling of a ship, Quart. J. Mech. Appl. Math. 1, 246-252 (1948) 